\title{
Environment-induced sudden transition in quantum discord dynamics
}

\author{
R. Auccaise, ${ }^{1}$ L. C. Céleri, ${ }^{2}$ D. O. Soares-Pinto, ${ }^{3}$ E. R. deAzevedo,${ }^{3}$ J. Maziero, ${ }^{2}$ A. \\ M. Souza, ${ }^{4}$ T. J. Bonagamba, ${ }^{3}$ R. S. Sarthour, ${ }^{5}$ I. S. Oliveira, ${ }^{5}$ and R. M. Serra ${ }^{2}$ \\ ${ }^{1}$ Empresa Brasileira de Pesquisa Agropecuária, Rua Jardim Botânico 1024, \\ 22460-000 Rio de Janeiro, Rio de Janeiro, Brazil \\ ${ }^{2}$ Centro de Ciências Naturais e Humanas, Universidade Federal do $A B C$, \\ R. Santa Adélia 166, 09210-170 Santo André, São Paulo, Brazil \\ ${ }^{3}$ Instituto de Física de São Carlos, Universidade de São Paulo, \\ Caixa Postal 369, 13560-970 São Carlos, São Paulo, Brazil \\ ${ }^{4}$ Fakultät Physik, Technische Universität Dortmund, 44221 Dortmund, Germany \\ ${ }^{5}$ Centro Brasileiro de Pesquisas Físicas, Rua Dr. Xavier Sigaud 150, \\ 22290-180 Rio de Janeiro, Rio de Janeiro, Brazil
}

\begin{abstract}
Non-classical correlations play a crucial role in the development of quantum information science. The recent discovery that non-classical correlations can be present even in separable (unentangled) states has broadened this scenario. This generalized quantum correlation has been increasing relevance in several fields, among them quantum communication, quantum computation, quantum phase transitions, and biological systems. We demonstrate here the occurrence of the suddenchange phenomenon and immunity against some sources of noise for the quantum discord and its classical counterpart, in a room temperature nuclear magnetic resonance setup. The experiment is performed in a decohering environment causing loss of phase relations among the energy eigenstates and exchange of energy between system and environment, resulting in relaxation to a Gibbs ensemble.
\end{abstract}

PACS numbers: 03.65.Yz, 03.65.Ud, 03.65.Ta

The quantum mechanical superposition principle, when applied to composite systems, foresees the appearance of correlations that cannot be explained in a classical context [1]. Initially, the quantum character of a correlated system was attributed to the nonlocal aspect of quantum mechanics and further associated with the violation of Bell's inequalities. The discovery of non-separable (entangled) states that do not violate the Bell's theorem led eventually to the identification of the quantumness of correlations with the separability problem; non-classicality was attributed to entanglement. Through the development of quantum information science (QIS), an operational characterization of entanglement was introduced, as those correlations that cannot be generated by local operations and classical communication [2]. The development of these ideas led to the so-called theory of entanglement, which turned out to be a fruitful branch of research [3. Entanglement is recognized as an important resource for several tasks in QIS [5]. Besides this strong correlation exhibited by entangled states, there is another kind of non-classical correlation. A composite quantum system in a mixed state may exhibit some non-classical nature in its correlations even if it is separable (unentangled) [5. Such quantum correlation can be measured as a "gap" between the quantum and classical versions of mutual information, which is the information-theoretic measure of the total correlation contained in a bipartite system [6].

Several approaches have been proposed to quantify this generalized quantum correlation present in a bipartite mixed quantum state [5, 7, 8], one of which, quantum discord [5], has received special attention [9 15. Beyond the importance of non-classical correlations (other than entanglement) for the foundations of quantum mechanics, the relevance of such an issue is increasing in several fields as well in applications in QIS. Concerning biological systems, it has been suggested that such correlations could play an important role in photosynthesis 9 . In condensed matter physics, quantum correlations of separable states characterize, even at finite temperature, a quantum phase transition [15. In the context of quantum field theory, it has been shown that the Unruh effect may also lead to an abrupt change in the behavior of correlations [16. For applications in QIS, it is interesting to know that states with nonzero quantum discord cannot be locally broadcast [17, and this kind of non-classicality is also related to a condition for a complete positive map [11. Quantum discord was proposed as a figure of merit for the quantum advantage in some computational models without or with little entanglement [12, 13. It also has a relevant role in mixed-state quantum metrology 18.

In particular, the quantum discord has been predicted to show peculiar dynamics under decoherence [6]. Considering two non-interacting qubits (quantum bits) under the action of Pauli maps, it was shown that, under certain conditions, the decay rate of the correlations suffers an abrupt modification, a phenomenon denominated sudden change [6. Moreover, either the classical or the quantum part of correlation may be unaffected by decoherence [6, 20, giving rise to two distinct decoherence regimes, the classical and the quantum. In the present 
experiment we demonstrate that the aforementioned phenomena [6, 20] are still present in a real thermal environment at room temperature, indicating that such peculiar behavior is quite general. We performed such experimental demonstration in a nuclear magnetic resonance (NMR) setup.

NMR systems have been extensively used as test benches for QIS ideas [21. Most of these experiments were performed in scenarios where the existence of entanglement was ruled out. The quantum nature of NMR systems at room temperature may be ascribed to quantum correlations of separable states [22]. The main feature of the technique for QIS is the excellent control of unitary transformations over the qubit provided by the use of radio-frequency pulses, which result in unique methods for quantum state generation and manipulation [21. In general, for room temperature liquid state NMR, the Markovian environment in which the qubit is immersed can be described by two decoherence channels, the amplitude-damping and the phase-damping, acting locally on each qubit. Our experiment is performed on a liquid state carbon-13 enriched chloroform sample at room temperature, this sample exhibits two qubits, encoded in the ${ }^{1} \mathrm{H}$ and ${ }^{13} \mathrm{C}$ spin- $1 / 2$ nuclei. The two-qubit state is represented by a density matrix in the high temperature expansion, which takes the form $\rho_{A B}=\mathbb{I}_{A B} / 4+\varepsilon \Delta \rho_{A B}$, where $\varepsilon=\hbar \omega_{L} / 4 k_{B} T \sim 10^{-5}$ is the ratio between the magnetic and thermal energies and $\Delta \rho_{A B}$ the deviation matrix [21, 25].

Several quantifiers of non-classical correlation have been proposed. From a thermodynamical approach, Oppenheim and coworkers have suggested that the nonclassical correlations contained in a bipartite quantum state can be quantified as the quantum deficit, a difference between the amount of work that can be extracted from a heat bath, by both and by one of the parts of the composite state [8]. In an attempt to build a unified framework for correlations, Modi and coworkers have proposed a set of measures for the quantification of their quantum and classical parts, based on the definition of an entropic distance [7. For the NMR context, one suitable generalized non-classical correlation may be computed from the experimentally accessible variables as a symmetric version of quantum discord [22, 24] (expanded in the leading order in $\varepsilon$ ), given by [22]

$$
Q\left(\rho_{A B}\right)=I\left(\rho_{A B}\right)-\max _{\left\{\Pi_{i}^{A}, \Pi_{j}^{B}\right\}} \mathcal{I}\left(\chi_{A B}\right),
$$

where the quantum mutual information is given by

$$
\begin{aligned}
& I\left(\rho_{A B}\right) \approx \frac{\varepsilon^{2}}{\ln 2}\left\{2 \operatorname{tr}\left(\Delta \rho_{A B}^{2}\right)-\operatorname{tr}\left[\left(\Delta \rho_{A}\right)^{2}\right]-\operatorname{tr}\left[\left(\Delta \rho_{B}\right)^{2}\right]\right\}, \\
& \text { and the measurement-induced } \quad \text { mu- } \\
& \text { tual information is } \mathcal{I}\left(\chi_{A B}\right) \\
& \frac{\varepsilon^{2}}{\ln 2}\left\{2 \operatorname{tr}\left[\left(\Delta \chi_{A B}\right)^{2}\right]-\operatorname{tr}\left[\left(\Delta \chi_{A}\right)^{2}\right]-\operatorname{tr}\left[\left(\Delta \chi_{B}\right)^{2}\right]\right\},
\end{aligned}
$$

with $\chi_{A B}=\mathbb{I}_{A B} / 4+\varepsilon \Delta \chi_{A B}$ as the state obtained from $\rho_{A B}$ through a complete projective measurement map $\left(\Delta \chi_{A B}=\sum_{i, j} \Pi_{i}^{A} \otimes \Pi_{j}^{B}\left(\Delta \rho_{A B}\right) \Pi_{i}^{A} \otimes \Pi_{j}^{B}\right)$. $\Delta \rho_{A(B)}=\operatorname{tr}_{B(A)}\left\{\Delta \rho_{A B}\right\}$ is the reduced deviation matrix while $\Delta \chi_{A(B)}$ stands for the reduced measured deviation matrix in the subspace $A(B)$. The classical counterpart of Eq. I1 is $C\left(\rho_{A B}\right)=\max _{\left\{\Pi_{i}^{A}, \Pi_{j}^{B}\right\}} \mathcal{I}\left(\chi_{A B}\right)$. It is worth mentioning that $Q\left(\rho_{A B}\right)=0$ if and only if $\rho_{A B}$ can be cast in terms of local orthogonal basis. In other words, the symmetric quantum discord is zero if and only if $\rho_{A B}$ has only classical correlations or no correlations at all 24]. The aforementioned symmetric correlation quantifiers can be computed directly from the experimentally reconstructed deviation matrix.

Let us consider the class of states with maximally mixed marginals $\left(\rho_{A(B)}=\mathbb{I}_{A(B)} / 2\right)$, also known as Belldiagonal states:

$$
\rho_{A B}=\frac{1}{4}\left(\mathbb{I}_{A B}+\sum_{i=x, y, z} c_{i} \sigma_{i}^{A} \otimes \sigma_{i}^{B}\right),
$$

where $\sigma_{i}^{A(B)}$ is the $i$ th component of the standard Pauli operator acting on the $A(B)$ subspace, $c_{i}=$ $\operatorname{tr}\left[\rho_{A B} \sigma_{i}^{A} \otimes \sigma_{i}^{B}\right]$, and $\mathbb{I}_{A B}$ is the identity operator. It was theoretically predicted [6] that, depending on the geometry (encoded in the relations between the parameters $c_{i}$ ) of the state (2), the evolution of the state correlations under the action of Pauli channels presents a peculiar behavior [6]. Considering the phase-damping channel, if the state components are related as follows: $\left|c_{z}\right| \geq\left|c_{x}\right|,\left|c_{y}\right|$, it was shown the classical correlation is not affected by decoherence, while the quantum correlation decays exponentially. On the other hand, if $\left|c_{x}\right| \geq\left|c_{y}\right|,\left|c_{z}\right|$ or $\left|c_{y}\right| \geq\left|c_{x}\right|,\left|c_{z}\right|$, and $\left|c_{z}\right| \neq 0$, the correlations exhibit the so-called sudden change in behavior [6]. The classical correlation decays exponentially until a specific moment in time, thereafter remaining constant, while the quantum correlation suddenly changes its decay rate at that moment. For the other two Pauli channels, we can see the same behavior, except that the relations between the three components of state (2) are exchanged [6].

In order to demonstrate such non-trivial dynamics we prepare two initial Bell-diagonal states with specific relations between its components. This involves the experimental mapping of the second term of the right hand side of Eq. (2) onto $\Delta \rho_{A B}$. Starting from the thermal equilibrium state, the mapping consists of three main steps, in which the following are applied to the sample: $(i)$ a strongly modulated pulse, (ii) a magnetic field gradient and (iii) a pseudo Einstein-Podolsky-Rosen gate implemented by radio-frequency pulses [21, 25]. After the preparation of the state, it is left to evolve freely under decoherence and the dynamics of the system is followed by quantum state tomography [3]. Figure 1 illustrates the pulse sequence in the experimental procedure [27. 


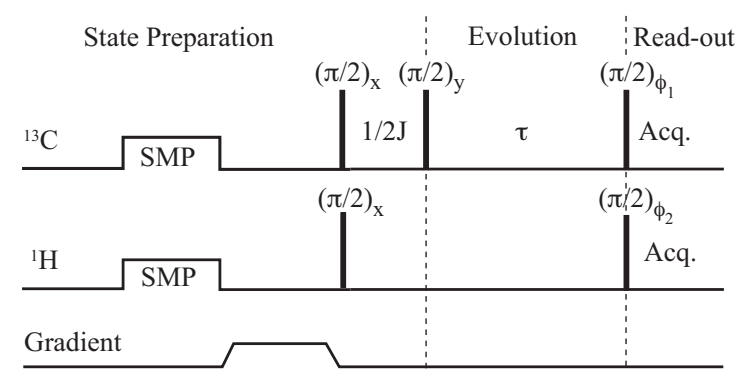

FIG. 1. Sketch of the pulse sequence used experimentally to follow the dynamics of quantum and classical correlations under decoherence. The sequence consists of three blocks: the initial state preparation, relaxation delay and read-out by quantum state tomography.

The relaxation process that causes phase decoherence and energy dissipation is due, mainly, to internal molecular or atomic motions that cause random fluctuations in the electromagnetic field in which the qubits are immersed. These fluctuations are characterized by the spectral densities that encode features of the motion, such as geometry and correlation times. The environment can be modeled by two independent quantum channels, described as the generalized amplitude-damping and the phase-damping channels. In previous investigations of sudden-change dynamics [6, 20, 23, the energy exchange channel was not take into account, however in the present experiment this relaxation source is unavoidable. It is remarkable that the sudden-change dynamics is still happen even in the presence of a thermal environment as can be seen in Fig. 2. The small decay rate of the classical correlation, after the transition point (where it could be constant under a phase damping), is due to the presence of the thermal noise source. The theoretical predictions presented in Fig. 2 are in good agreement with the experimental data [27. We observe a sudden transition between two decoherence regimes [6], i.e., classical and quantum decoherence [20]. During the first regime $(t \lesssim 0.027 \mathrm{~s})$ the decoherence affects more strongly the classical aspects of correlation, leaving the quantum aspects with a small decay. After the sudden-change point $(t \gtrsim 0.027 \mathrm{~s})$ the classical correlation becomes more robust against decoherence and the noise degrades more effectively the quantumness of correlations. This confirms that the phenomena predicted in [6, 20, for phase environments takes place even in the presence of an additional thermal noise.

Figure 3 also confirms the theoretical prediction [6] that, in some states (depending on their geometry), the classical correlation may be robust against phase noise. The small decay rate of this correlation plotted in Fig. 3 comes entirely from the thermal source. Once more, the theoretical curves presents very good agreement with the experimental data.

In both of the Figs. 2(c) and 3(c), the theoretical data
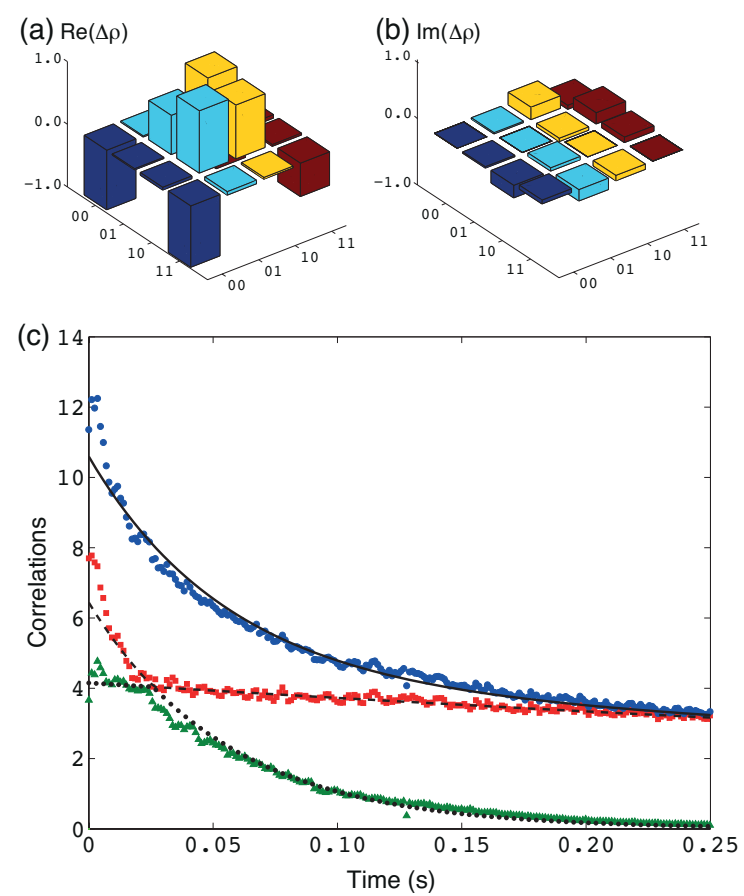

FIG. 2. (Color online) Sudden change in behavior of correlations. (a) bar representation of the real and, (b) imaginary parts of the initial deviation matrix for the sudden-change experiment, reconstructed by quantum state tomography. We adopted the usual computational basis, where $|0\rangle$ and $|1\rangle$ represents the eigenstates of $\sigma_{z}$ for each qubit. (c) displays the predicted sudden change in behavior of the correlations during their dynamic evolution to thermal equilibrium. The blue circles are the experimental data for the quantum mutual information, while the red squares and green triangles represent the classical and quantum correlations, respectively. The black lines are the theoretical predictions. The initial state is analogous to the state in Eq. (2) with $\left|c_{x}\right|,\left|c_{y}\right|>\left|c_{z}\right|$. The correlations are displayed in units of $\left(\varepsilon^{2} / \ln 2\right)$ bit.

analysis guarantees that the small decay rate presented by the classical correlation, where it should vanish, comes exclusively from the action of the thermal channel on the system. It is worth mentioning that the oscillations shown by the experimental curves in both figures are due to experimental details (discussed in [27]).

Correlations are ubiquitous in nature. The discovery that separable quantum states can exhibit non-classical correlation other than entanglement has led to a new understanding of the quantum aspects of a physical system. Despite the relatively great number of theoretical articles concerning decoherence effects, up to date, only few experimental investigations of the correlation dynamics have been reported previously in literature [22, 23, 28]. The predictions of refs. [6, 20] were tested in a simulated phase noise environment [23]. This experiment was performed in an optical setup, in which the action of a phase-damping channel was simulated in a controllable way by a birefringent medium and the qubits were en- 

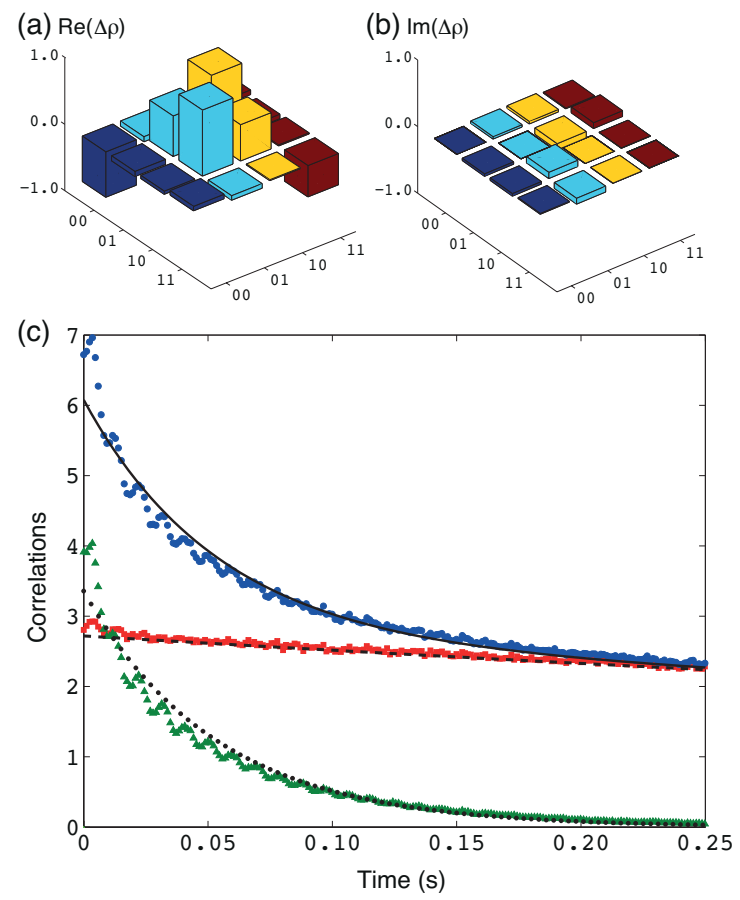

FIG. 3. (Color online) Immunity against decoherence. (a) bar representation of the real and, (b) imaginary parts of the initial deviation matrix, reconstructed by quantum state tomography. (c) shows that the classical correlation is not affected by the action of the phase-damping channel. The small decay rate is entirely due to the amplitude-damping channel. The displayed pattern is the same as in Fig. 2. For this experiment, the initial state is analogous to state in Eq. (2) with $\left|c_{z}\right|>\left|c_{x}\right|,\left|c_{y}\right|$. The correlations are computed in units of $\left(\varepsilon^{2} / \ln 2\right)$ bit.

coded in photon polarizations [23]. Under the action of a real global environment, the dynamics of correlations was investigated in a NMR quadrupolar system [22].

In the present experiment we observed the environment-induced sudden change phenomena in a real (uncontrollable) thermal noise environment at room temperature. The environment-induced sudden change takes place in the course of the relaxation of two nuclear spins to the Gibbs state. This demonstrate that this phenomena may occur in a general context when a non-equilibrium system relax to a thermal equilibrium state. The methods employed in our experiment to follow the dynamics of quantum discord and its classical counterpart may be applied to other molecules (including biological ones) and also to other experimental contexts where high mixed states are present. The two decoherence regimes observed may have important consequences for applications in QIS, since the nature of correlations in a given mixed state system is somehow related to the quantum advantage for performing a given task (as for example in quantum metrology [18]) or preventing local broadcasting of information [17.

The authors acknowledge financial support from
UFABC, CNPq, CAPES, FAPESP, and FAPERJ. This work was performed as part of the Brazilian National Institute of Science and Technology for Quantum Information (INCT-IQ).

[1] J. S. Bell, Speakable and Unspeakable in Quantum Mechanics (Cambridge University Press, Cambridge, 1988).

[2] R. F. Werner, Phys. Rev. A 40, 4277 (1989).

[3] R. Horodecki et al., Rev. Mod. Phys. 81, 865 (2009).

[4] M. A. Nielsen and I. L. Chuan, Quantum Computation and Quantum Information (Cambridge University Press, Cambridge, 2000).

[5] H. Ollivier and W. H. Zurek, Phys. Rev. Lett. 88, 017901 (2001).

[6] B. Groisman, S. Popescu, and A. Winter, Phys. Rev. A 72, 032317 (2005).

[7] K. Modi et al., Phys. Rev. Lett. 104, 080501 (2010);

[8] J. Oppenheim et al., Phys. Rev. Lett. 89, 180402 (2002).

[9] K. Brádler et al., Phys. Rev. A 82, 062310 (2010).

[10] R. Dillenschneider and E. Lutz, Europhys. Lett. 88, 50003 (2009).

[11] A. Shabani and D. A. Lidar, Phys. Rev. Lett. 102, 100402 (2009).

[12] A. Datta, A. Shaji, and C. M. Caves, Phys. Rev. Lett. 100, 050502 (2008).

[13] B. P. Lanyon et al., Phys. Rev. Lett. 101, 200501 (2008).

[14] D. Cavalcanti et al., Phys. Rev. A 83, 032324 (2011); V. Madhok and A. Datta, Phys. Rev. A 83, 032323 (2011).

[15] J. Maziero et al., Phys. Rev. A 82, 012106 (2010); T. Werlang et al., Phys. Rev. Lett. 105, 095702 (2010); J. Maziero et al., e-print arXiv:1012.5926 (2010).

[16] L. C. Céleri et al., Phys. Rev. A 81, 062130 (2010).

[17] M. Piani, P. Horodecki, and R. Horodecki, Phys. Rev. Lett. 100, 090502 (2008).

[18] K. Modi et al., e-print arXiv:1003.1174 (2010).

[19] J. Maziero et al., Phys. Rev. A 80, 044102 (2009).

[20] L. Mazzola, J. Piilo, S. Maniscalco, Phys. Rev. Lett. 104, 200401 (2010).

[21] I. S. Oliveira, T. J. Bonagamba, R. S. Sarthour, J. C. C. Freitas, E. R. deAzevedo, NMR Quantum Information Processing (Elsevier, Amsterdam, 2007).

[22] D. O. Soares-Pinto et al., Phys. Rev. A 81, 062118 (2010).

[23] J.-S. Xu. et al., Nat. Commun. 1, 7 (2010).

[24] J. Maziero, L. C. Céleri, and R. M. Serra, e-print arXiv:1004.2082 (2010).

[25] L. M. K. Vandersypen, and I. L. Chuang, Rev. Mod. Phys. 76, 1037 (2004).

[26] G. L. Long, H. Y. Yan, Y. Sun, J. Opt. B: Quantum Semiclass. Opt. 3, 376 (2001); J. Teles et al., J. Chem. Phys. 126, 154506 (2007).

[27] See supplementary material for a detailed description of the experimental and theoretical procedures.

[28] R. Auccaise et al., e-print arXiv:1104.1596 (2011). 


\section{Supplementary information - Details on experimental and calculation procedures}

\section{NMR experiments}

NMR experiments were performed on a two-quantum bit system composed of nuclear spins of ${ }^{1} \mathrm{H}$ and ${ }^{13} \mathrm{C}$ atoms in carbon- 13 enriched chloroform $\left(\mathrm{CHCl}_{3}\right)$. The sample was prepared by mixing $100 \mathrm{mg}$ of $99 \%{ }^{13} \mathrm{C}$ labelled $\mathrm{CHCl}_{3}$ in $0.2 \mathrm{~mL}$ of $99.8 \% \mathrm{CDCl}_{3}$ in a $5 \mathrm{~mm}$ NMR tube (both compounds provided by the Cambridge Isotope Laboratories Inc.). NMR experiments were carried out at $25^{\circ} \mathrm{C}$ in a Varian $500 \mathrm{MHz}$ Infinity Plus spectrometer located at the Brazilian Center for Physics Research (CBPF, Rio de Janeiro). A Varian $5 \mathrm{~mm}$ double resonance probehead equipped with a magnetic field gradient coil was used. $\pi / 2$ pulses of length $7.4 \mu \mathrm{s}$ and $8.0 \mu$ s were applied to ${ }^{1} \mathrm{H}$ and ${ }^{13} \mathrm{C}$, respectively. For specific state preparation, a five-steps strongly modulated pulses (SMP) 1] was designed using a MATLAB self-written routine based on a SIMPLEX optimization protocol. Spin-lattice relaxation times $\left(\mathrm{T}_{1}\right)$ for ${ }^{1} \mathrm{H}$ and ${ }^{13} \mathrm{C}$ nuclei, measured by the inversion recovery pulse sequence, were $2.5 \mathrm{~s}$ and $7 \mathrm{~s}$, respectively. Spin-spin relaxation times $\left(\mathrm{T}_{2}\right)$, measured by a CPMG pulse sequence, were estimated to be about $1.8 \mathrm{~s}$ and $0.29 \mathrm{~s}$ for ${ }^{1} \mathrm{H}$ and ${ }^{13} \mathrm{C}$ nuclei, respectively. The recycle delay $\left(\mathrm{d}_{1}\right)$ was set at $40 \mathrm{~s}$ in all experiments. As no refocusing pulse was used, the effective transversal relaxation times are given by $T_{2}^{*}=0.31 \mathrm{~s}$ and $T_{2}^{*}=0.12 \mathrm{~s}$ for ${ }^{1} \mathrm{H}$ and ${ }^{13} \mathrm{C}$ nuclei, respectively.

The nuclear spin Hamiltonian accounting for the relevant NMR interactions is given by

$$
\begin{aligned}
\mathcal{H}= & -\left(\omega_{H}-\omega_{r f}^{H}\right) \mathbf{I}_{z}^{H}-\left(\omega_{C}-\omega_{r f}^{C}\right) \mathbf{I}_{z}^{C}+2 \pi J \mathbf{I}_{z}^{H} \mathbf{I}_{z}^{C} \\
& +\omega_{1}^{H}\left(\mathbf{I}_{x}^{H} \cos \varphi^{H}+\mathbf{I}_{y}^{H} \sin \varphi^{H}\right)+\omega_{1}^{C}\left(\mathbf{I}_{x}^{C} \cos \varphi^{C}+\mathbf{I}_{y}^{C} \sin \varphi^{C}\right),
\end{aligned}
$$

where $\mathbf{I}_{\alpha}^{H}\left(\mathbf{I}_{\beta}^{C}\right)$ is the spin angular momentum operator in the $\alpha, \beta=x, y, z$ direction for ${ }^{1} \mathrm{H}\left({ }^{13} \mathrm{C}\right) ; \varphi^{H}\left(\varphi^{C}\right)$ defines the direction of the RF field (pulse phase) and $\omega_{1}^{H}\left(\omega_{1}^{C}\right)$ is the RF nutation frequency (RF power) for ${ }^{1} \mathrm{H}\left({ }^{13} \mathrm{C}\right)$ nuclei. The first two terms describe the Zeeman interaction between the ${ }^{1} \mathrm{H}$ and ${ }^{13} \mathrm{C}$ nuclear spins and the main magnetic field $\mathrm{B}_{0}$. The corresponding frequencies are $\omega_{H} / 2 \pi \approx 500 \mathrm{MHz}$ and $\omega_{C} / 2 \pi \approx 125 \mathrm{MHz}$. The third term is due to a scalar spin-spin coupling of $J \approx 215.1 \mathrm{~Hz}$. The fourth and fifth terms represent the radio-frequency field (RF) that may be applied to the ${ }^{1} \mathrm{H}$ and ${ }^{13} \mathrm{C}$ nuclear spins, respectively. Besides the interactions written above, there is a time-dependent coupling of the nuclear spins with the environment that includes all fluctuating NMR interactions, which accounts for the spin relaxation, e.g., ${ }^{1} \mathrm{H}-{ }^{13} \mathrm{C}$ dipolar spin-spin couplings, interactions with the chlorine nuclei, etc.

To follow the decoherence of the correlations and distinguish the distinct regimes described in the main text, an initial state such as that shown in Eq. (1) of the the main text needs to be prepared with specific $c_{i}$ 's . Therefore, from the experimental perspective, it is essential to prepare a state in which populations and coherences of the deviation matrix can be set accordingly. To that end, a diagonal state was created from the thermal equilibrium by a $4-8 \mathrm{~ms}$ SMP followed by a $2 \mathrm{~ms}$ magnetic field gradient pulse. This produced a deviation matrix as shown in Eq. (4), where the elements $\alpha, \beta, \gamma$ and $\delta$ are defined by the optimized SMP pulse. Next, this diagonal deviation matrix is transformed into an X-type matrix, such as that in Eq. (5), by a pseudo-EPR gate imple- mented by the RF pulse sequence described in reference [2].

$$
\begin{aligned}
\Delta \rho & =\left[\begin{array}{llll}
\alpha & 0 & 0 & 0 \\
0 & \beta & 0 & 0 \\
0 & 0 & \gamma & 0 \\
0 & 0 & 0 & \delta
\end{array}\right], \\
\Delta \rho & =\frac{1}{2}\left[\begin{array}{cccc}
\alpha+\gamma & 0 & 0 & -\alpha+\gamma \\
0 & \beta+\delta & -\beta+\delta & 0 \\
0 & -\beta+\delta & \beta+\delta & 0 \\
-\alpha+\gamma & 0 & 0 & \alpha+\gamma
\end{array}\right] .
\end{aligned}
$$

The pulse sequence used in the NMR experiments is illustrated in Fig. 1 of the main text. The SMP was designed to produce a diagonal state with populations $\alpha, \beta, \gamma$ and $\delta$, so that, after the transformation by the pseudo-EPR gate, a state with a deviation matrix in the form equivalent to the state in Eq. (2) of the main text, with specified $c_{i}$ 's, was obtained. After this state preparation step, the system was left to evolve for a period $\tau=m / 4 J$, with $m=1,2,3,4, \ldots$ During this evolution period (considering on-resonance evolution), two terms of the Hamiltonian shown in Eq. (3) act on the nuclear spins: the scalar coupling, which produces oscillations of the quantum coherence, and the time-dependent term, which accounts for the system relaxation. It is worth mentioning that the elements in the main and cross diagonals of the deviation matrix account for populations and quantum coherences that do not oscillate with the $J$ coupling evolution, so that, for a perfect X-type deviation matrix, the aforementioned oscillation should not be 
observed. However, the experimental deviation matrices have small coherences off the $\mathrm{X}$ positions, which oscillate during the evolution. This leads to small oscillations in the spectral line intensities used to perform the state tomography and, because the tomography process involves solving coupled equations, this oscillatory behaviour is captured in the main and cross diagonal elements, causing the oscillations observed in the correlations shown in Figs. 2 (c) and 3 (c) of main text. The oscillatory evolution may be suppressed from the experimental data by setting the evolution step to a multiple of $1 / J$. However, in order to show unequivocally the sudden change in the quantum correlations, the evolution step was set to $1 / 4 J$, giving rise to the small oscillations observed in the evolution of the correlations depicted in Figs. 2 (c) and 3 (c) of the main text. After the evolution period, the quantum state was read-out by quantum state tomography, as described in reference [3, 4. Multi-step increments of $\tau$ in successive experiments allowed the effect of the spin environment on the initial deviation matrix to be followed. Final states corresponding to 251 distinct $\tau$ values were acquired. The high homogeneity of the static magnetic field was guaranteed by the linewidth of $\approx 0.95 \mathrm{~Hz}$ in ${ }^{1} \mathrm{H}$ spectra.

\section{Decoherence analysis}

The decoherence process is theoretically analysed through the operator sum representation technique [5], in which the evolution of the density operator is given by

$$
\rho(t)=\sum_{k} E_{k}(t) \rho(0) E_{k}^{\dagger}(t),
$$

where $E_{k}(t)$ are the well-known Kraus operators.

Amplitude-Damping Channel: In this scenario, the amplitude-damping channel is described by the following set of operators

$$
\begin{aligned}
& E_{0}=\sqrt{\gamma}\left(\begin{array}{cc}
1 & 0 \\
0 & \sqrt{1-p}
\end{array}\right), \quad E_{1}=\sqrt{\gamma}\left(\begin{array}{cc}
0 & \sqrt{p} \\
0 & 0
\end{array}\right), \\
& E_{2}=\sqrt{1-\gamma}\left(\begin{array}{cc}
\sqrt{1-p} & 0 \\
0 & 1
\end{array}\right), \quad E_{3}=\sqrt{1-\gamma}\left(\begin{array}{cc}
0 & 0 \\
\sqrt{p} & 0
\end{array}\right),
\end{aligned}
$$

where, in the NMR context, $\gamma=1 / 2-\varepsilon / 2$ and $p=$ $1-\exp \left(-t / T_{1}\right), T_{1}$ being the longitudinal relaxation time of the qubit under consideration. We observe that in our case, the relaxation times are different for the two qubits, since they have distinct Larmor frequencies.

Phase-Damping Channel: For this case we have

$$
E_{0}=\sqrt{1-\frac{\lambda}{2}}\left(\begin{array}{ll}
1 & 0 \\
0 & 1
\end{array}\right), \quad E_{1}=\sqrt{\frac{\lambda}{2}}\left(\begin{array}{cc}
1 & 0 \\
0 & -1
\end{array}\right),
$$

where $\lambda=1-\exp \left(-t / T_{2}\right)$ with $T_{2}$ being the transverse relaxation time associated with the qubit.

The agreement between the model and the experimental data, as observed in Figs. 2 (c) and 3 (c) of the main text, is fairly good. In the theoretical model we neglected a number of possible error sources, such as magnetic field inhomogeneities or oscillations of the residual off-diagonal elements of the deviation matrix. In spite of that, all the singular features predicted by the quantum model are very clear in the experimental data.

Within this model and taking into account that both environments act independently on each qubit, it is straightforward to eliminate from the experimental data the contribution of the amplitude-damping channel to the dynamics of the correlations. This procedure removes the small decay rate of the classical correlation (which it should be constant) in both Figs. 2 (c) and 3 (c) of the main text, proving that this correlation is really immune to the phase-damping channel in certain cases, corroborating the theoretical predictions made in ref. [6].

[1] E. M. Fortunato et al. J. Chem. Phys. 116, 7599 (2002).

[2] I. L. Chuang, N. Gershenfeld, M. G. Kubinec, and D. W. Leung Proc. R. Soc. A 454, 447 (1998).

[3] G. L. Long, H. Y. Yan, and Y. Sun, J. Opt. B: Quantum Semiclass. Opt. 3, 376 (2001).

[4] J. Teles, E. R. deAzevedo, R. Auccaise, R. S. Sarthour, I. S. Oliveira, and T. J. Bonagamba, J. Chem. Phys. 126, 154506 (2007).

[5] M. A. Nielsen and I. L. Chuang, Quantum Computation and Quantum Information (Cambridge University Press, Cambridge, 2000).

[6] J. Maziero, L. C. Céleri, R. M. Serra, and V. Vedral, Phys. Rev. A 80, 044102 (2009). 